\title{
A Case of Oral leukoplakia Recovered by Receiving HRT for 3 Months
}

\author{
Kiyomitsu Miyachi $^{1 *}$, Asuka Imanishi ${ }^{2}$, Atsushi Watanabe ${ }^{3}$, Akiko Ihara ${ }^{1}$, Sosei Kou $^{4}$ and \\ Takao Koyama ${ }^{5}$ \\ ${ }^{1}$ Higashiterao Second Clinic, Japan \\ ${ }^{2}$ Tsurumi University Dental Hospital, Japan \\ ${ }^{3}$ Asunaro Pharmacy, Japan
}

${ }^{4}$ Kou Ladies Mental Clinic Enoshima, Japan

${ }^{5}$ Koyama Takao Clinic, Japan

*Corresponding author: Kiyomitsu Miyachi, Rheumatology, Women's health and aging, Keigu Clinic, Yokohama, Japan.

To Cite This Article: OKiyomitsu Miyachi*, Asuka Imanishi, Atsushi Watanabe, Akiko Ihara, Sosei Kou, et al., A Case of Oral leukoplakia Recovered by Receiving HRT for 3 Months. Am J Biomed Sci \& Res. 2019 - 1(4). AJBSR.MS.ID.000539. DOI: 10.34297/AJBSR.2019.01.000539

Received: February 24, 2019 | Published: March 06, 2019

\begin{abstract}
Leucoplakia is well known as a pre-malignant disease in oral tumour. About 80 years ago, hormone replacement therapy was useful for the treatment of oral leucoplakia. However, no report regarding efficacy of HRT has not been obtained since then.

Case $48 \mathrm{y}$ female, past history: 20y ovarian cyst, no smoking. She became menopause in 2014 and noticed dry mouth and oral white coating without pain. She visited at oral surgery of Tsurumi Dental University and was diagnosed as a leukoplakia, following biopsy. Oral and dental examination revealed no evidence of apparent causes and she received dental care, although no efficacy. She visited Keigu clinic, complaining of shoulder stiffness and pain, palpitation and dry mouth in April 2017. Her sex hormone level showed E2 $12.0 \mathrm{pg} / \mathrm{mL}$, FSH 83.7mIU/mL, suggesting loss of her ovarian function. Immunological examination showed CRP $0.10 \mathrm{mg} / \mathrm{dl}, \mathrm{RF}<4$ and ANA $<40$. She has no possibility of having autoimmune rheumatic disease. She began receiving HRT and was surprised at disappearing of leucoplakia for 3 months. She keeps in good health until the end of 2018 under HRT receiving.
\end{abstract}

Keywords: Leukoplakia; HRT; Oral tumor

\section{Introduction}

Leukoplakia appears in the oral mucosa, gingiva and tongue and the main body of the lesion is stratified squamous epithelium, dyskeratosis. Male is twice as large as female and age of onset is 50 to 70's. It is thought that this disease is a pre-cancer state in which cancer is transferred to 5 to $10 \%$ [1]. Before 1940, there was a report on efficacy of HRT for leukoplakia [2]. We report one case in which hormone replacement therapy (HRT) was effective for intractable lesion

Results

i. $\quad$ Case: 48-year-old female, 1973

ii. Family history: No special mention

iii. Past history: 1973 Ovarian cyst, 2014 menopause, no smoking

\section{Current Medical History}

Morning stiffness and right shoulder pain were started in 2014 and subsequently, she went to menopausal stage and developed dry mouth. There was a burning white patch without pain on the oral mucosa in September 2014. In Tsurumi Dental University Hospital, she got a diagnosis of leukoplakia by oral biopsy. Although the cause remained unknown. Oral care was given but it did not feel better. In July 2017, she visited Keigu Clinic with shoulder joint pain, palpitations, dry mouse etc. Low level of E2 $12.0 \mathrm{pg} / \mathrm{mL}$ and high level of FSH 83.7 mIU / mL meant ovarian dysfunction.

\section{Laboratory findings and clinical course (Table 1)}

In the course of 1 year and 8 months from July 2017 to February 2019, both rheumatoid factor (RF) and anti-CCP antibody were negative. Moreover, escalation of CRP and joint swelling were 
not noted. Arthralgia is rapidly alleviated by HRT. In addition, ANA determination revealed neither anti-SS-A antibody (Ab) nor
anti-nRNP Ab. HRT was administered from September 2017 and leukoplakia disappeared in 3 months.

\begin{tabular}{|c|c|c|c|c|c|c|c|c|}
\hline & 2017.7 & 2017.8 & 2017.9 & 2017.11 & 2017.12 & 2018.4 & 2018.9 & 2019.2 \\
\hline WBC & 6000 & & 5600 & & & 4900 & 4900 & 4200 \\
\hline Plt & 21.3 & & 25.1 & & & 17.7 & 19.3 & 21.2 \\
\hline CRP & 0.1 & & & & & 0.04 & 0.09 & 029 \\
\hline RA & 4 & & & & & 4 & 4 & 4 \\
\hline ACPA & 2) & & & & & & & \\
\hline ANA & & & $40>$ & & & & & \\
\hline staining pattern & & & ro & & & & & \\
\hline anti-SSA & & & 2 & & & & & \\
\hline E2 & 12 & & & & & 25.1 & 50.1 & 109.7 \\
\hline $\mathrm{FSH}$ & 83.7 & & & & & 63.3 & 71.5 & 39.5 \\
\hline $\begin{array}{l}\text { joint tenderness } N \\
\text { joint swelling } N\end{array}$ & 5 & & 0 & & & & & 0 \\
\hline RVAS & 100 & & & 21 & & 10 & & 10 \\
\hline ERT & & E2mono & & & & & & \\
\hline EPRT & & & \multicolumn{6}{|c|}{ EPRT cyclic } \\
\hline Leukcplakia & & & & & & & & \\
\hline
\end{tabular}

\section{Leukoplakia on left buccal mucosa (Figure 1)}

The third molars and the next are filled with metal and adjacent these the white patch and linear were seen on the left oral mucosal area.

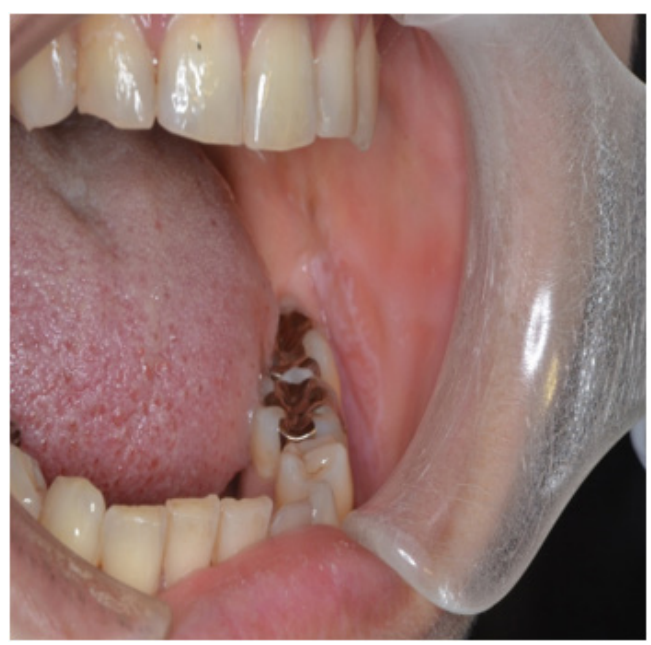

Figure 1: Leukoplakia on left buccal mucosa.

Histological findings by biopsy of right oral mucosa (Figure 2)

The specimen biopsied from the right buccal mucosal part is covered with a stratified squamous epithelium which partially elongated irregular epithelium leg with accompanying parakeratosis. Under the epithelium chronic inflammatory cells mainly lymphocytes are intermittently observed in focal (Figure 2a).

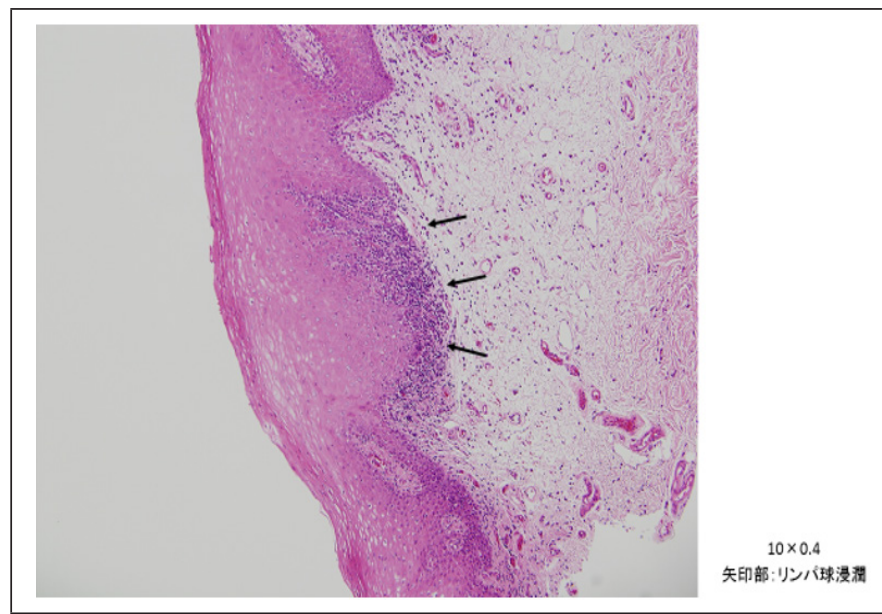

Figure 2a: Histological findings by biopsy of right oral mucosa at low magnification.

In the same area, liquid degeneration of the basal layer was also observed, possibilities of lichen planus were considered, but on this section the part showing characteristic findings was extremely localized, so it was diagnosed as leukoplakia (Figure 2b). 


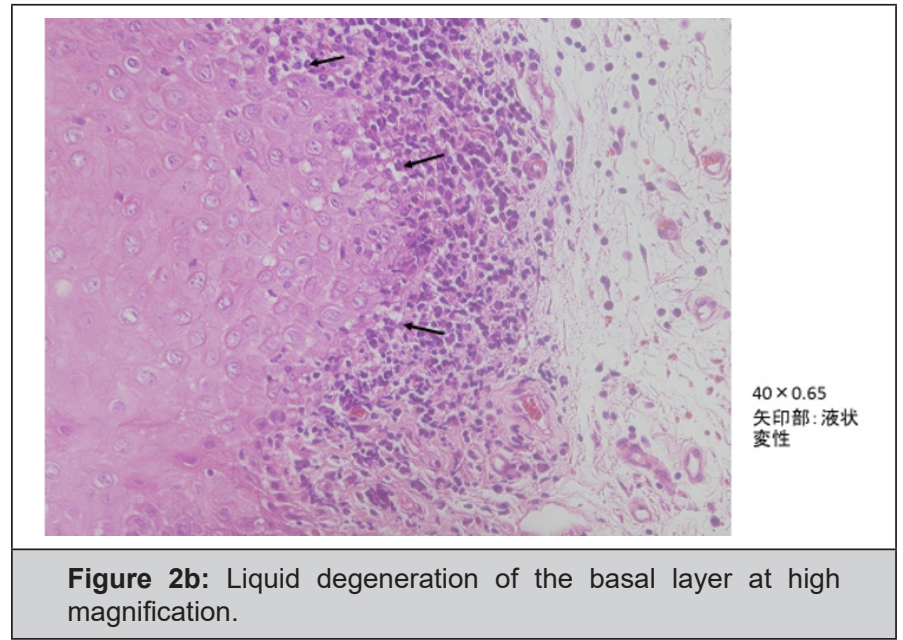

Discussion

We presented a peri- to post-menopausal women having oral leucoplakia which was healed by HRT for 2 months. Recent treatment of leucoplakia included exclusion of white patch by operation, taking vitamin A, chemical substance, and estrogen (HRT). However, we could not find any report of efficacy of HRT since 1938.

Peri- and post-menopausal women have visited clinics having of many complaints such as hot flush, palpitation, insomnia, depression, morning stiffness, and peripheral joint pains, lipidoses [3], appearing ANA [4]. Hormone replacement therapy (HRT) was prescribed in these patients, subsequently they got good quality of life. In the recent, I have experienced postmenopausal women complaining of shoulder pain and dry mouth on the first visit. In this case, both RF and anti-CCP Ab were negative, moreover both elevation of CRP and joint swelling were not noted, she was not diagnosed as having RA but postmenopausal arthralgia, because HRT leaded to more $70 \%$ of reduce joint pain [5].

Her oral leukoplakia was accidentally disappeared following HRT for three months. Leukoplakia of the oral mucosa is white patch or plaque which cannot be removed by friction, and it is defined as being unable to explain in other diseases. Little leukoplakia is reported to be somewhat higher in elderly from the middle age, but it was also reported in the past that it is common in middle - aged women [2]. Although there are many uncertainties in the cause, there are reports of teeth, allergies of packing materials, smoking, etc. There was a report that the balance of sex hormones has collapsed in the past [2]. In the study on this case, a parakeratosis of the stratum corneum, stratification of the stratum corneum, liquefaction of the basal layer was confirmed by oral biopsy histopathological examination. There were no cases reporting the effectiveness of hormone replacement therapy (HRT) for leukoplakia. It seemed to me it just happened that HRT was effective. To our surprise, the effectiveness of HRT for leukoplakia was reported from 1935 to 1939 [2]. The content of the paper was the administration of 0.5 to $1 \mathrm{mg}$ of $\alpha$ - estradiol between 21 and 150 days, 16 out of 38 cases (42\%) disappeared, 15 cases (39\%) improved, and 7 cases (19\%) did not improve, which was a shocking result. In addition, the effect of male is weak. When it recurs after discontinuation, it is easy to relieve by re-administration.

If it is administered earlier, it improves easily. The case of severe cases takes time to improve. It is found that administration of estradiol is essential. Indeed, this case was diagnosed as leukoplakia during the transition period of menopause, white patch occurred for at least 3 years, became menopause and disappeared in 3 months after HRT was performed at our clinic. There are few reports on treatment of leukoplakia in Japanese reports. Ogawa and colleagues reported 34 cases, but the ratio between male and female was $17: 17$, there was no gender difference, $50 \%$ in the 50 s and 60 s, accounting for leukoplakia causes filling materials 14, unknown 14, abnormal teeth 5, estrogen deficiency 1 [6]. One patient had HRT for 13 years, but it became leukoplakia after discontinuation. Although the cause of leukoplakia is complicated, hormone replacement therapy seems to be the first choice of treatment when it appears in relatively young women. However, it is difficult for dentists and oral surgeons to implement HRT, so cooperation with gynecologists is desirable. I hope that this report again examines the effect of HRT on the precancerous symptom with leukoplakia.

Finally, we don't know why HRT regresses leukoplakia in this patient. It is possible that HRT persistently suppresses the NFkB activation leading to pre-cancer tumor such as leukoplakia [7]. Subsequently, oral leukoplakia region would be disappeared. However, more extensive study will be needed.

\section{Acknowledgement}

We thank Prof. Hamada Yoshiki at the Department of Oral surgery and Prof. Itou Yoshimi at the Department of Pathology in Tsurumi Dental University Hospital.

\section{References}

1. Jolán Bánóczy, Zeno Gintner, Csaba Dombi (2001) Tobacco Use and Oral Leukoplakia. Journal of Dental Education 65(4): 322-327.

2. Nathanson IT, Weishberger DB (1939) The treatment of leukoplakia buccalis and related lesion with estrogenic hormone. N Engl J Med 221: 556-561.

3. Miyachi K, Ihara M, Ishikawa T, Sasse B, Igarashi T, et al. (2018) Two Long-Term Follow-Up Cases Preventing Atherosclerosis by Hormone Replacement Therapy-CETP Deficiency and Tangier Disease. Biomed J Sci \&Tech Res 9(5)

4. Miyachi K, Ihara A, Ihara M, Yamamoto K, Sasse B, et al. (2018) AntiDFS70 Autoantibodies ln Japanese Women at Different Menstrual Stages. Journal of Rheumatology Research 1(1): 24-32.

5. Miyachi K, Sasse B, Nomoto S, Igarashi T, Mashiba S, et al. (2019) The Treatment of Women with Post-Menopausal Undifferentiated Arthralgia -The First Report of Efficacy of Hormone Replacement Therapy. American Journal of Biomedical Science \& Research 1: 124-129.

6. Ogwa T, Tanio K, Ryoke K, Hamada T (1983) Clinical and HistoPathological Studies on the Cases of Leukoplakia Experienced in Past 15 years. J Yonago Med Ass 34: 273-284.

7. Murphy AJ, Guyre PM, Pioli PA (2010) Etradiol Suppresses NF-kappa B activation through Coordinated Regulation of Let-7a and MiR-125b in Primary Human Macrophages. J Immunol 184(9): 5029-5037. 